tional und erlebniszentriert tätigen Therapeuten, für die dieses Buch geschrieben ist, Weiterbildungen bei körperorientierten, psychotherapeutischen Methoden - und damit ist sicherlich die Konzentrative Bewegungstherapie, die Integrative Bewegungstherapie, Tanztherapie, Körperpsychotherapie und weitere Methoden gemeint - auf ein großes Interesse stoßen (S. 21). Und ich stimme der Auffassung der Autoren vollumfänglich zu, dass eine Doppelqualifikation Bewegungs- / PsychotherapeutIn die klinische Versorgung sicherlich bereichern kann. Um die Kritik auch mal umzudrehen: Auch bei KörperpsychotherapeutInnen werden funktional übungszentrierte Techniken und Methoden wie Lauftherapie und Ausdau- ertherapie, z.B. bei Depression und Schmerz, nach wie vor zu wenig eingesetzt.

Das Autorenteam Thimme, Deimel und Hölter legt wirklich ein fundiertes, differenziertes und akribisch ausgearbeitetes Werk zur motorisch-funktionellen und erlebniszentrierten Bewegungstherapie für Kinder und Jugendliche auf dem neuesten Wissenschaftsstand vor. Es wird die Bewegungstherapie bekannter machen, und man kann es allen Fachgruppen, die mit Kindern und Jugendlichen arbeiten, sehr empfehlen.

Martin J. Waibel

DOI 10.2378/ktb2021.art21d

\title{
Jonas A. Hamm: Trans* und Sex. Gelingende Sexualität zwischen Selbstannahme, Normüberwindung und Kongruenzerleben
}

Psychosozial-Verlag, 2020, Gießen, 147 Seiten, 19,90€ (D)

\section{L} ängst überfällig, dieses Buch! Was Jonas A. - Hamm unter gelingender Sexualität in seinem Buch „Trans* und Sex“ aufschlüsselt, ist für viele Menschen noch nicht selbstverständlich und somit ein enorm wichtiger Beitrag zur Ent-Tabuisierung von (Trans*-)Sexualität. Fein und ausdifferenziert beobachtend zeigt Hamm die große Lücke auf zwischen gesellschaftlicher Realität, wissenschaftlicher Aufarbeitung und persönlicher Erfahrung von gelebter Sexualität. Das realisiert er über eine qualitative Studie mit sechs sorgfältig ausgewählten und reflektierten Personen einer Minderheit, die die sehr konkrete, sexuelle Realität von Trans*Personen zeigt, welche keine genitalangleichende/n Operation/en anstreben.

Sowohl für mich persönlich, der auch zu dieser Minderheit gehört, als auch für viele meiner KlientInnen hat dieses Buch die Bedeutung eines Meilensteins in seiner Perspektive von / auf und für Trans*-Men-

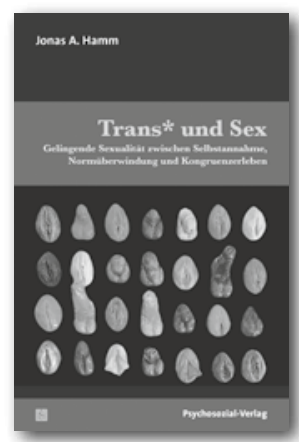
schen mit dem Ziel ihrer Selbstbestimmung über den eigenen Körper.

Als systemischer Berater, Somatiker und Sexological Bodyworker begleite ich Trans*Menschen darin, ihre Verbindung zum eigenen Körper zu stärken. Dabei ist meine Erfahrung, dass es besonders unterstützend ist, vor allem die Aspekte der gelingenden Sexualität in den Fokus gestellt zu wissen. Diese, wie in diesem Werk noch mal ausdifferenziert betrachtet, schaffen unter anderem Anregung zur Selbstreflexion, sind aber vor allem für die Begleitprozesse in Bezug auf eigene internalisierte Vorstellungen wertvoll. Die Grundfrage „Was brauchst du, um mit deiner eigenen Sexualität zufrieden zu sein, mit deinem eigenen Körper 
aktiv zu sein, fernab von normativen und vorgeschriebenen Zuschreibungen, die gesellschaftlich existieren in Bezug auf Körper, Identität, Gender und Begehren?" bildet eine deutliche Ausgangsperspektive. Als lesende Person darf ich den einzelnen Personen in den sexuellen Profilen nahekommen, darf an ihrer wundervollen Sexualität und an ihren Lernund Reflexionsprozessen teilhaben.

Durch die partizipative Feedbackschleife gelingt es dem Autor, mit den Menschen zu sprechen und mit ihnen zu schreiben anstatt über sie, was Augenhöhe und Vertrauen und ein sehr respektvolles Setting mit ausgewogenen Anteilen von Leading und Pacing schafft. Gleichzeitig überzeugt die überaus systemische Haltung mit dem Fokus auf der individuellen Sexualität sowie auf den Strategien und Ressourcen und dem direkt angebundenen sexuellen und geschlechtlichen Lernprozess jeder teilnehmenden Person. In ihren sexuellen Profilen sprechen diese offen über ihr Begehren und ihre Art und Weise, Sex zu haben. Sie thematisieren ihre Vorlieben, wie sie mit ihren Genitalien umgehen, Toys verwenden und ihren ganzen Körper einsetzen. Und sie beschreiben, wie sie in Kommunikation darüber mit ihren Sexualpartnerlnnen sind. Ich bin Hamm sehr dankbar für diese respektvolle und außerordentlich bereichernde Darlegung in Schriftform.

Für die Gesundheitsversorgung aller Menschen wünsche ich mir, dass zukünftig die Perspektive des Gelingens mehr gewichtet wird als die des Nicht-Gelingens und der bislang noch häufig daraus resultierenden, keineswegs hilfreichen Konsequenz von Pathologisierung.

Und wie auch Annette Güldenring in ihrem Geleitwort schreibt „Die Suche nach kongruenten Begegnungen der Liebe ist für alle Menschen eine große Aufgabe“, so denke ich, dass es keinen Weg gibt außer dem direkten. Es geht darum, sich selbst kennenzulernen und zu erforschen und sich immer weiter abzugrenzen von existierenden normativen Bildern und Vorstellungen von Sexualität. Von Trans*-Menschen können Cis-Menschen, also Menschen, deren Geschlechtsidentität mit dem Geburtsgeschlecht übereinstimmt, noch viel lernen. Dieses Buch geht hierfür einen weiteren Schritt voran.

Nino Mar Seliz

DOI 10.2378/ktb2021.art22d 